\title{
Bit-Allocation Strategies for MIMO Fading Channels with Channel Knowledge at Transmitter
}

\author{
Joon Hyun Sung and John R. Barry \\ School of Electrical and Computer Engineering \\ Georgia Institute of Technology, Atlanta, Georgia 30332-0250 \\ \{jhsung, barry\}@ece.gatech.edu
}

\begin{abstract}
The singular-value decomposition can be used to transform a MIMO fading channel into an equivalent bank of scalar subchannels, a strategy known as eigenbeamforming or closed-loop MIMO, provided that the transmitter knows the channel. We consider the problem of allocating bits to subchannels after such processing, and propose simple strategies, which achieve near-optimal performance, by exploiting statistical properties of singular values. For large antenna arrays, a fixed bit-allocation becomes an attractive choice without any significant performance loss. For example, on the 6-input 6-output Rayleigh-fading channel, the fixed allocation strategy performs only $0.25 \mathrm{~dB}$ worse than the optimal bit-allocation.
\end{abstract}

\section{INTRODUCTION}

For a bank of scalar channels, it is well-known that capacity is achieved by water-pouring procedures when channel information is known to the transmitter [1]. In practice, where rates are often restricted to be finite and discrete values, the problem must be modified, to account for the granularity constraint.

The best way to allocate discrete rates to parallel channels would compare all possible combinations of allocations and select the best one. However, this exhaustive search often requires high complexity and thus iterative optimization is widely used instead. Many iterative algorithms for allocation have been introduced, especially for discrete multitone (DMT) applications, such as [2]-[4], which have relatively small complexity without significant performance loss.

For MIMO channels, parallel subchannels are created by a singular-value decomposition (SVD) (see [5] and references therein). Unlike DMT, MIMO flat-fading channels have some unique properties: the number of subchannels in MIMO systems is small compared to

This research is supported by the Yamacraw initiative, http://wwwyarnacraw.org.
DMT, and the singular values of channel matrices, which are the subchannel gains, exhibit special properties. In this paper, we study the bit-allocation problem using the special properties of MIMO channels.

The rest of this paper is organized as follows. Section II describes the MIMO flat-fading model and the problem statement. In Section III, we propose lowcomplexity bit-allocation strategies, and explore their near-optimal performance. Performance is evaluated for Rayleigh fading in Section IV. In Section V, we examine the robustness of the proposed strategies to a change of the fading statistics. Finally, we conclude in Section VI.

\section{System Model and Bit-Allocation Problem}

For simplicity we consider a narrowband channel with $M$ transmit and $M$ receive antennas, which can be modeled by an $M \times M$ channel matrix $\mathbf{H}=\left[h_{i j}\right]$, where $h_{i j}$ is the response at receive antenna $i$ from transmit antenna $j$. Let $\mathbf{H}=\mathbf{U d i a g}\left(\boldsymbol{s}^{1 / 2}\right) \mathbf{V}^{*}$ denote an SVD, where $\mathbf{U}$ and $\mathbf{V}$ are unitary, and where the elements of $s=\left[s_{1}, \ldots, s_{M}\right]$ are real and ordered so that $s_{1} \geq \ldots \geq s_{M} \geq 0$. When the transmitter and receiver filter by $\mathbf{V}$ and $\mathbf{U}^{*}$, respectively, a bank of scalar subchannels results:

$$
y_{i}=\sqrt{s_{i}} a_{i}+n_{i}, \quad \text { for } i=1, \ldots M,
$$

where $\left\{n_{i}\right\}$ are i.i.d. $\mathcal{C}\left(0, N_{0}\right)$. There is no crosstalk from one subchannel to the next.

So as to achieve a rate of $r_{i}$ bits per signaling interval across the $i$-th subchannel, its SNR $s_{i} E_{i} / N_{0}$ must be at least $\Gamma\left(2^{r_{i}}-1\right)$, where $E_{i}=E\left[\left|a_{i}\right|^{2}\right]$, and where $\Gamma$, an SNR gap, accounts for the additional SNR required for a practical code to achieve a given target probability of error [2]. With an ideal capacity-achieving code, $\Gamma$ reduces to unity. Then the total energy, $\Sigma_{i} E_{i}$, required by the transmitter to achieve a given set of rates $\left\{r_{i}\right\}$ is:

$$
E(s)=\Gamma \sum_{i=1}^{M} \frac{2^{r_{i}}-1}{s_{i} / N_{0}} .
$$


It is well-known that, to achieve a given total rate of $\boldsymbol{R}=$ $\sum_{i} r_{i}$ bits per signaling interval, the rate allocation that minimizes (2) is given by the water-pouring solution, $r_{i}=\left\{\log _{2}\left(\lambda(s) s_{i} / \Gamma\right)\right\}^{+}$, where $\{x\}^{+}=\max \{0, x\}$, and where $\lambda(s)$ ensures that $R=\sum_{i} r_{i}$.

In practice, complexity considerations require that $\left\{r_{i}\right\}$ be drawn from a discrete and finite set. Let the granularity, $\beta$, be the smallest incremental unit of information rate. Then, the rate of any subchannel is given by $r_{i}=\beta B_{i}$, where $B_{i}$ is a non-negative integer. With these constraints, the bit-allocation problem is to find the $\left\{r_{i}\right\}$, given $s$, that minimizes (2) subject to a total rate constraint, $R=\sum_{i} r_{i}$. Clearly, the best bit-allocation is based on a full search that enumerates each element of:

$\mathcal{B}=\left\{\left[r_{1}, \ldots, r_{M}\right] ; \sum_{i} r_{i}=\beta \Sigma_{i} B_{i}=R, r_{1} \geq \ldots \geq r_{M} \geq 0\right\},(3)$

and chooses the allocation that minimizes (2) for given 8 . The ordering restriction on $\left\{r_{i}\right\}$ in (3) stems from the ordered nature of $s$. When $M$ is large, such as in DMT, the size of full-search set $(\mathcal{B})$ can be very large and calculating (2) for all members in $\mathcal{B}$ might be practically too complex. Even for MIMO flat-fading channels, where the number of subchannels is not as large as DMT due to physical space limitation of antenna arrays, the complexity of full-search strategy can be high. Usually, full-search strategy seems to be feasible only for $M=2$. In the paper, we consider how to reduce the complexity without any significant performance loss.

\section{Proposed Bit-Allocation Strategy}

First we investigate how frequently an allocation in the full-search set is used and how it contributes to average required SNR, when channels, $\left\{h_{i j}\right\}$, are generated according to a certain distribution, such as Rayleigh distribution. Let $b_{j}=\left[b_{1 j}, \ldots, b_{M j}\right]$ be the $j$-th allocation in $\mathcal{B}$ and let $\boldsymbol{A}_{j}$ be a subregion in $\boldsymbol{M}$-dimensional space, $\{\boldsymbol{s}$; $\left.s_{1} \geq \ldots \geq s_{M} \geq 0\right\}$, in which $b_{j}$ is optimal. In other words, if $s \in A_{j}, b_{j}$ is the bit-allocation that minimizes (2) with $r_{i}$ $=b_{i j}$ for $i=1, \ldots M$. Then, the average required SNR for a full search becomes:

$$
E / N_{0}=\mathrm{E}_{A_{j}}\left[\mathrm{E}_{\mathbf{s}}\left[\Gamma \sum_{i=1}^{M} \frac{2^{r_{i}}-1}{s_{i}} \mid A_{j}\right]\right]=\sum_{j=1}^{L} P_{j} \varepsilon_{j},(4)
$$

where $\varepsilon_{j}=\Gamma \sum_{i}\left(2^{R_{c} b_{i j}}-1\right) \mathrm{E}_{g}\left[1 / s_{i} \mid A_{j}\right]$ is the partial SNR requirement conditioned on $A_{j}$, and where $L$ denotes the size of $\mathcal{B}$. The probability mass function (PMF) of allocation is denoted by $P_{j}=\operatorname{Prob}\left[A_{j}\right]$ for $j=1, \ldots L$, which indicates how often $\boldsymbol{b}_{j}$ is selected over realizations of $\boldsymbol{s}$.
One way to reduce search-set size is based on the following observations. For given fading statistics, some elements of $\mathcal{B}$ are infrequently or never used, that is, $\boldsymbol{P}_{j}$ is very small or zero for some $j$.

Observation 1. If $P_{j}$ is negligible, deleting its allocation $b_{j}$ from $B$ and using other allocation(s) for $A_{j}$ will increase $E / N_{0}$, but its increase is only marginal.

This is obvious since small $P_{j}$ nults the increase in $P_{j} \varepsilon_{j}$ by using suboptimal allocation for $A_{j}$. Hence, deleting these infrequent allocations from consideration has little impact on performance.

In order to see how deleting members from $\mathcal{B}$ impacts performance, we investigate the increase in average SNR by deleting members one by one. We delete the allocation that has the smallest $P_{j}$, and calculate corresponding average SNR penalty compared to full-search strategy. We repeat these procedures until all but one allocation are eliminated. Fig. 1-a illustrates average SNR penalty in $\mathrm{dB}$ as allocations are removed from $\mathcal{B}$ for $\beta=3 / 4$ and $B=12$, where we assume $B_{i} \in\{0, \ldots 8\}$ in (3) and, where $10^{5}$ independent Rayleigh channels $(M=6)$ are generated. As illustrated in Fig. 1-b, which plots $P_{j}$ for $j=$ $1, \ldots L$, there are seven allocations out of $L=51$ which have dominant $P_{j}$. Labels in Fig. 1-b identify seven dominant- $P_{j}$ allocations, and the impact of eliminating them is shown in Fig. 1-a, where alphabetical order matches deletion order. It can be seen in Fig. 1-a that penalty is almost zero until seven labeled allocations are left in reduced set, which agrees with Observation 1. By removing the ' $a$ ' allocation, SNR penalty begins to grow sharply, and the last elimination (labeled as ' $f$ ') leaves only one allocation (labeled as 'survivor') in the search set.

An interesting point in this elimination process is how many allocations have negligible $\boldsymbol{P}_{j}$, so that they do not affect (4) much. As observed in Fig. 1-b, only a few out of $L$ possibilities have dominant $P_{j}$, and thus the number of considerations reduces correspondingly.

Observation 2. In MIMO channels the number of allocations with dominant $P_{j}$ is small relatively to the size of full-search set, $L$.

This can be explained in part by ordered natures of singular values and reduced variability of bit-allocation. By ordered nature, we mean not only that singular values are ordered, $s_{1} \geq \ldots \geq s_{M}$, but also that each singular value has a different distribution. Thus, depending on the distributions of the singular values, some allocations have 
(a)

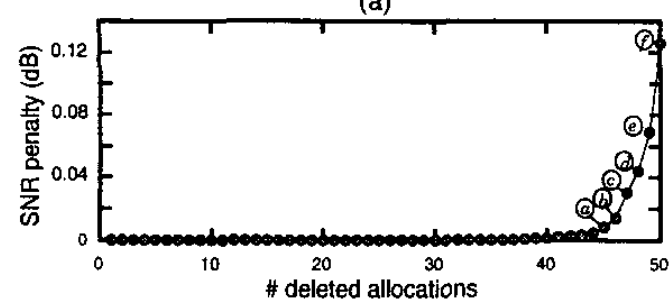

(b)

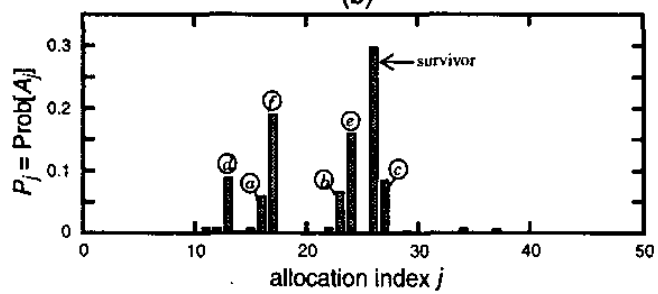

Fig. 1. (a) SNR penalty in $\mathrm{dB}$ due to deleting bit-allocations from $\mathcal{B}$. (b) PMF $\left(P_{j}\right)$ of bit-allocations, both for $M=6$ and $B=12$.

higher probabilities than others, which implies that the bit-allocation is more predictable. Certainly, there is stil variability in bit-allocation despite the ordered nature. The point is how small this variability is. To this purpose, we investigate distribution of the optimal rate, $r_{i}=\left\{\log _{2}\left(\lambda(s) s_{i} / \Gamma\right)\right\}^{+}$. For instance, Fig. 2 illustrates empirical marginal distribution of optimal rate for $M=4$ (thick) and $M=6$ (thin) at $R / M=2$ bits per signaling interval and per antenna, where we assume $\mathrm{E}\left[\left|h_{i j}\right|^{2}\right]=1$. Even though the marginal distribution tells only a part of the whole story, a variability reduction is obvious as one goes from $M=4$ to $M=6$ in Fig. 2. In $M$-dimensional space, we conjecture, from marginal distribution and covariance of optimal rates, that only a small portion of space corresponds to large probability as joint distribution is centered and has small variance (in all directions), and that distribution shrinks, that is, variance becomes small for large $M$. This conjecture suggests that only a few allocations in $\mathcal{B}$, whose $A_{j}$ corresponds to high-probability regions of optimal rates, have dominant $P_{j}$.

Now we move further and delete some of the allocations with dominant $P_{j}$, as inspired from Fig. 1-a, where deleting all but one allocation incurs only a penalty of $0.13 \mathrm{~dB}$ compared to full search.

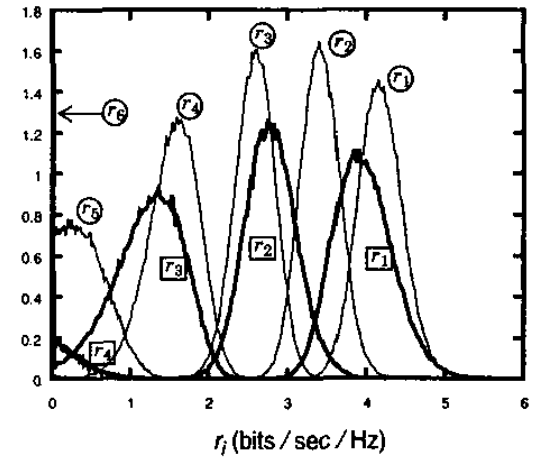

Fig. 2. Marginal PDF of optimal rate for $M=4$ (thick) and for $M=6$ (thin) at $R / M=2$ bits $/ \mathrm{sec} / \mathrm{Hz} /$ antennas.

Observation 3. A penalty by removing $b_{j}$ with dominant $P_{j}$ is not negligible any more, but still reasonably small.

This is based on the fact that the increase in $\varepsilon_{j}$ in (4) is small, even if $P_{j}$ is not negligible, since distance between the optimal $\boldsymbol{b}_{j}$ and its substitute is not quite far as regions of frequently-used allocations concentrate in $\boldsymbol{M}$ dimensional space.

From Observations 1-3, we propose bit-allocation strategies restricting its search to $\mathcal{B}_{1}$ and $\mathcal{B}_{2}$, where $\mathcal{B}_{k}$ denotes a restricted search set containing only $k$ candidate allocations. For optimal choice of $\mathcal{B}_{k}$, we compare average required SNR for all possible members of $\mathcal{B}_{k}$, and choose the one that produces minimum average required SNR. For most cases, the optimal choice coincides with the results by deleting infrequently-used allocation one by one as in Fig. 1-a, but it is not always true, especially when $M=2$.

Advantages of proposed strategies include: (i) a great reduction in complexity; (ii) thus suitable to frequent channel change; (iii) no increase in complexity as number of subchannels $(M)$ grows; and (iv) applicable to any constraint on rate (e.g. any stepsize $\beta$ or any maximum value of $B_{i}$ ). Complexity reduction is quite impressive when compared to a full search. For example, only two calculations of (2) are required if $\mathcal{B}_{2}$ is used, in contrast to 51 calculations required for a full search when $M=6$ and $B=12$ for $B_{i} \in\{0, \ldots 8\}$. Especially low in complexity is 


\begin{tabular}{|c|c|c|}
\hline \multicolumn{3}{|c|}{$M=2$} \\
\hline & $B_{2}$ & $\mathcal{B}_{1}$ \\
\hline$B=2$ & {$\left[\begin{array}{lll}20 & 0\end{array}\right],\left[\begin{array}{lll}1 & 1\end{array}\right]$} & [20] \\
\hline$B=4$ & {$\left[\begin{array}{ll}40],[31] \\
\end{array}\right.$} & [4 0] \\
\hline$B=6$ & $\{60\},[51\}$ & [60] \\
\hline$B=8$ & $[80] .62]$ & {$[80]$} \\
\hline
\end{tabular}

\begin{tabular}{|c|c|c|}
\hline \multicolumn{3}{|c|}{$M=4$} \\
\hline & $B_{2}$ & $\mathcal{B}_{1}$ \\
\hline$B=4$ & 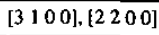 & {$[3100]$} \\
\hline$B=8$ & 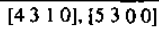 & {$\left[\begin{array}{lllll}4 & 3 & 1 & 0\end{array}\right]$} \\
\hline$B=12$ & {$\left[\begin{array}{lllll}5 & 43 & 0\end{array},\left[\begin{array}{lll}6 & 4 & 2\end{array}\right]\right.$} & $\{6420\}$ \\
\hline$B=16$ & $[7540], 17630]$ & $17630]$ \\
\hline
\end{tabular}

\begin{tabular}{|c|c|c|}
\hline \multicolumn{3}{|c|}{$M=6$} \\
\hline & $\mathcal{B}_{2}$ & $\mathcal{B}_{1}$ \\
\hline$B=4$ & 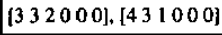 & {$\left[\begin{array}{llllll}4 & 3 & 1 & 0 & 0 & 0\end{array}\right]$} \\
\hline$B=12$ & 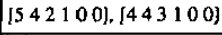 & {$\left[\begin{array}{llllll}5 & 4 & 2 & 1 & 0 & 0\end{array}\right]$} \\
\hline$B=18$ & {$[653200],[554200]$} & 16532001 \\
\hline$B=24$ & {$\left[\begin{array}{ll}65432 & 20] .[754310]\end{array}\right.$} & {$\left[\begin{array}{lll}7 & 543 & 10]\end{array}\right.$} \\
\hline
\end{tabular}

Fig. 3. Samples of restricted sets $\left(\mathcal{B}_{1}\right.$ and $\left.\mathcal{B}_{2}\right)$ optimized to Rayleigh for $M \in\{2,4,6\}$.

restricting search to $\mathcal{B}_{1}$ (fixed allocation), which does not require bit-allocation processing. This will be particularly valuable when these ideas extend to frequency-selective channels.

As mentioned before, on the other hand, choice of $\mathcal{B}_{1}$ and $\mathcal{B}_{2}$ depends on channel distribution. Thus, we assume that channel statistics as well as channel information are known to transmitter. If $\mathcal{B}_{1}$ and $\mathcal{B}_{2}$ do not match current fading statistics, it could cause significant performance loss. In Section V, we will deal with this mismatch problem.

\section{Numerical Results}

We consider Rayleigh fading for $M \in\{2,4,6\}$ antennas. Suppose that each allocation is restricted to discrete values, $r_{i}=\beta B_{i}$, with $\beta=0.75$ and $B_{i} \in\{0, \ldots 8\}$. Fig. 3 illustrates restricted-search sets, $\mathcal{B}_{1}$ and $\mathcal{B}_{2}$, optimized for Rayleigh fading, for some $B=\sum_{i} B_{i}=R / \beta$. In Fig. 3, for example, an allocation denoted as [4 2] means $B_{1}=4$ and $B_{2}=2$. Notice that, in the case of $\mathcal{B}_{1}$, the last subchannel $\left(s_{M}\right)$ is never used. This is because $s_{M}$ is exponentially distributed in Rayleigh fading [6], which means that, information-theoretically, it takes infinite average power to convey a nonzero rate, however small it is, over this subchannel. Especially for $M=2$, all information is forcefully conveyed over the first subchannel, which explains why optimal allocation is not necessarily most frequently-used allocation, and which inevitably results in a significant performance loss.
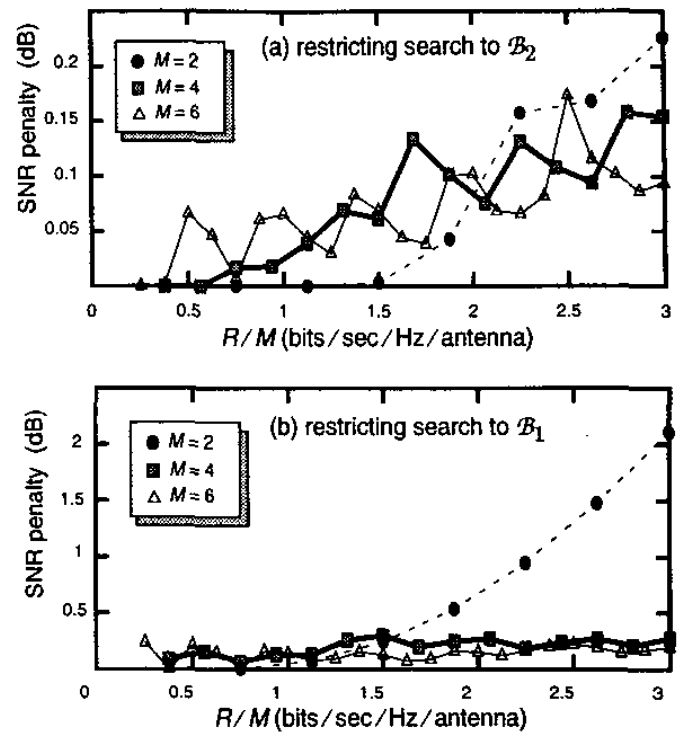

Fig. 4. Relative performance of restricted search over $\mathcal{B}_{1}$ in (a) and over $\mathcal{B}_{2}$ in (b) compared to full-search strategy in Rayleighfading with $M \in\{2,4,6\}$.

We evaluates performance of restricted search over $\mathcal{B}_{1}$ and $\mathcal{B}_{2}$ in Fig. 3 , which plots average SNR penalty by using $\mathcal{B}_{2}$ in Fig. 4-a, and by using $\mathcal{B}_{1}$ in Fig. 4-b against rate per signaling interval and per antenna when compared to full-search strategy $(\mathcal{B})$. In both cases, the restricted searches perform only marginally worse. For example, restricting the search to $\mathcal{B}_{2}$ incurs an SNR penalty of less than $0.23 \mathrm{~dB}$ when $M=2$. Even the fixed allocation $\left(\mathcal{B}_{1}\right)$ performs well, falling only $0.3 \mathrm{~dB}$ short of the full-search performance for both $M=4$ and $M=6$. One exception is for $M=2$, where the penalty by using $\mathcal{B}_{1}$ can be as large as $2.2 \mathrm{~dB}$ at $R / M=3$.

\section{ROBUST BIT-Allocation STRATEgy}

As discussed at the end of Section III, bit-allocation based on restricted search over $\mathcal{B}_{k}$ could cause a mismatch problem when actual fading statistics are different from those to which $\mathcal{B}_{k}$ is optimized. When size of $\mathcal{B}_{k}$ is small, this mismatch problem can be serious. In the following we consider mild mismatch, which occurs when statistics estimation differs from real channels or when statistics slightly change between estimations. Also we focus on the case of more than two antennas $(M>2)$. 
An intuitive way to make bit-allocation robust to fading change is to increase the size of $\mathcal{B}_{k}$ and to select its members appropriately. Since restricting search to $\mathcal{B}_{1}$ is near-optimal for $M>2$, an union of several $\mathcal{B}_{1}$ 's, whose members are optimized to some typical fading statistics, would perform reliably. Expanding $\mathcal{B}_{k}$ obviously leads to increase in complexity. For mild change of statistics, however, only a few additions are sufficient.

For example, consider Ricean fading of $K=4.45$ in $M$ $=4$ antenna arrays with $\beta=0.75$ and $B_{i} \in\{0, \ldots 8\}$, where $K$ denotes the Rice factor [7]. The dotted line in Fig. 5 represents SNR penalty by using a mismatched $\mathcal{B}_{2}$, which is optimized to Rayeligh ( $K=0$ ), compared to a full search over $\mathcal{B}$. This mismatch loss can be large, as illustrated in Fig. 5, more than $1 \mathrm{~dB}$. By adding three $\mathcal{B}_{1}$ 's that are optimized to $K=0, K=2.41$, and $K=6.46$, respectively, we constitute a robust search set:

$$
\mathcal{B}_{3, \text { rob }}=\mathcal{B}_{1, K=0} \cup \mathcal{B}_{1, K=2.41} \cup \mathcal{B}_{1, K=6.46},
$$

where $\mathcal{B}_{1, K=6.46}$ means that $\mathcal{B}_{1}$ is optimized to $K=6.46$. Fig. 5 shows performance of robust bit-allocation strategy, where thick line corresponds to $\mathcal{B}_{3, \text { rob }}$. As references, SNR penalty of $\mathcal{B}_{1}$ (square) and $\mathcal{B}_{2}$ (circle), which are optimized to actual fading $(K=2.41)$, is plotted. Clearly, bit-allocation over $\mathcal{B}_{3 \text {,rob }}$ performs very well, whose SNR penaity is less than $0.2 \mathrm{~dB}$.

\section{CONCLUSIONS}

Based on the statistical properties of MIMO fading channels, we proposed reduced-complexity bit-allocation strategies that restrict the search to $\mathcal{B}_{2}$ (containing two allocations) or $\mathcal{B}_{1}$ (fixed allocation). These bit-loading strategies considerably reduce complexity while performing only marginally worse than optimal bitallocation. For example, in Rayleigh fading with $M=4$ or $M=6$ antennas, its average SNR penalty is below 0.15 $\mathrm{dB}$ and below $0.3 \mathrm{~dB}$ when restricting search to $\mathcal{B}_{2}$ and $\mathcal{B}_{1}$, respectively. For $M=2$, it has been found that at least two allocations must be considered $\left(\mathcal{B}_{2}\right)$. We also proposed a robust bit-allocation strategy which can handle some variations of fading statistics.

The proposed bit-allocation strategies extend to orthogonal frequency division multiplexing (OFDM) in frequency-selective channels. If each OFDM tone is restricted to have the same bit-budget, a great deal of complexity can be saved by the proposed strategies. Since frequency correlation between tones is ignored, it would incur a performance loss. Future work will compare the flat-frequency strategy with conventional bit-allocation

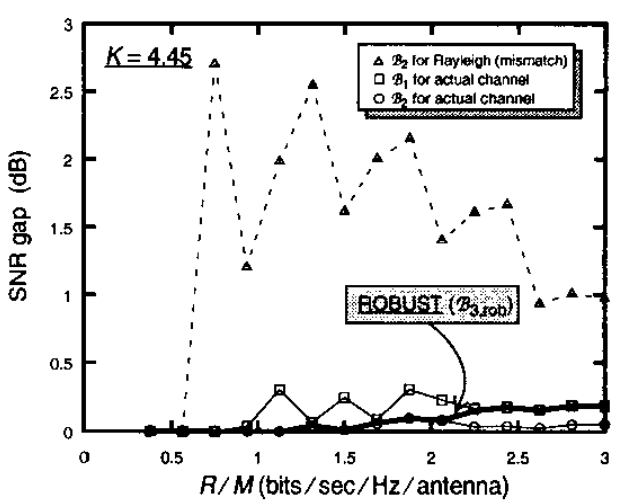

Fig. 5. Fading mismatch of restricted search over $\mathcal{B}_{2}$ optimized to Rayleigh fading and performance of robust bit-allocation in Ricean fading ( $K=4.45)$ with $M=4$ antennas.

algorithms. In the meantime, we only consider a fixed total rate system in this paper. We will investigate joint optimization of bit-allocation and rate regions when the total rate is variable.

\section{REFERENCES}

[1] R. M. Cover. and J.A. Thomas, Elements of Infornation Theory, Wiley \& Sons. 1991

[2] J. M. Cioffi, Lecture Notes for Advanced Digital Communications. Standford. 2002

[3] P. S. Chow, J. M. Cioffi, and J. A. C. Bingham. "A practical discrete multitone transceiver loading algorithm for data transmission over spectrally shaped channels," IEEE Trans. Commun., vol. 43, pp. 773-775. Feb. 1995.

[4] J. Campello, "Practical bit-loading for DMT," IEEE ICC 1999, Vancouver. Canada, vol. 2, pp. 801-805. 1999.

[5] J. H. Sung, and J. R. Barry. "Space-time processing with channel knowledge at the transmitter," EUROCON 2001, Bratislava. Slovakia, vol. 1, pp. 26-29, Jul. 2001.

[6] A. Edelman, "Eigenvalues and condition numbers of random matrices." $P$ h.D. Dissertation, Massachusetts Institute of Technology, 1989.

[7] G. L. Stuber, Principles of Mobile Communication, Kluwer Academic Publishers, 1996. 